\title{
Structural Basis of RICs Iron Donation for Iron-Sulfur Cluster Biogenesis
}

\author{
Liliana S. O. Silva1', Pedro M. Matias ${ }^{1,2}$, Célia V. Romão ${ }^{1 *}$ and Lígia M. Saraiva ${ }^{1 *}$ \\ 1 Instituto de Tecnologia Química e Biológica António Xavier, Universidade Nova de Lisboa, Oeiras, Portugal, ${ }^{2}$ iBET, Instituto \\ de Biologia Experimental e Tecnológica, Oeiras, Portugal
}

Escherichia coli YtfE is a di-iron protein of the widespread Repair of Iron Centers proteins $(\mathrm{RIC})$ family that has the capacity to donate iron, which is a crucial component of the biogenesis of the ubiquitous family of iron-sulfur proteins. In this work we identify in E. coli a previously unrecognized link between the YtfE protein and the major bacterial system for iron-sulfur cluster (ISC) assembly. We show that YtfE establishes proteinprotein interactions with the scaffold IscU, where the transient cluster is formed, and the cysteine desulfurase IscS. Moreover, we found that promotion by YtfE of the formation of an Fe-S cluster in IscU requires two glutamates, E125 and E159 in YtfE. Both glutamates form part of the entrance of a protein channel in YtfE that links the di-iron center to the surface. In particular, E125 is crucial for the exit of iron, as a single mutation to leucine closes the channel rendering YtfE inactive for the build-up of Fe-S clusters. Hence, we provide evidence for the key role of RICs as bacterial iron donor proteins involved in the biogenesis of Fe-S clusters.

Keywords: di-iron protein, iron-sulfur biosynthesis, protein-protein interaction, site-direct mutagenesis, X-ray crystallography

\section{INTRODUCTION}

The Repair of Iron Centers proteins (RIC) is a family of di-iron proteins that provide protection to enzymes prone to inactivation by oxidative and nitrosative stresses imposed by the host's innate immune system (Justino et al., 2006, 2009; Overton et al., 2008). Firstly discovered in Escherichia coli, YtfE is a member of the RIC family present in bacteria, fungi and eukaryotes (Justino et al., 2005; Overton et al., 2008). In particular, homologs of these proteins are encoded in the genomes of a significant number of human pathogens, such as Bacillus anthracis, Haemophilus influenzae, and species of the genus Salmonella, Shewanella, Yersinia, or Clostridium. Two RIC paralogs (RIC1 and RIC2) are also present in the eukaryote Trichomonas vaginalis, an important human pathogenic protozoan (Nobre et al., 2016). In vivo studies showed that RIC deletion in E. coli and Staphylococcus aureus generates strains defective in the activity of several ironsulfur-containing proteins, and RICs contribute to survival and virulence of S. aureus, Yersinia pseudotuberculosis, and H. influenzae (Overton et al., 2008; Harrington et al., 2009; Silva L.O. et al., 2018; Davis et al., 2019).

Bacterial RICs are composed by an $\mathrm{N}$-terminal ScdA-like domain and a $\mathrm{C}$-terminal domain that folds as a four-helix bundle where a histidine/carboxylate di-iron type center is inserted. In E. coli YtfE, the best studied RIC protein, the center is coordinated by H84, H129, H160, H204, 
and two $\mu$-carboxylate bridges formed by E133 and E208 (Nobre et al., 2015). These residues are highly conserved in the RIC family and are required for the assembly of a stable and functional di-iron cluster (Nobre et al., 2015). Mössbauer and EPR studies showed that the center of the as-isolated YtfE has properties characteristic of a mixed valence antiferromagnetically coupled $\mathrm{Fe}(\mathrm{III})-\mathrm{Fe}(\mathrm{II})$ center with a $\mathrm{S}=1 / 2$ ground state (Todorovic et al., 2008; Nobre et al., 2014, 2015). Mössbauer studies further revealed that one of the iron atoms in the center is labile, particularly in the mixed-valence $\mathrm{Fe}(\mathrm{III})-\mathrm{Fe}$ (II) state when compared with the $\mu$-oxo-diferric form $\mathrm{Fe}(\mathrm{III})-\mathrm{Fe}$ (III) (Nobre et al., 2014). In the reduced form, YtfE $\mathrm{Fe}(\mathrm{II})-\mathrm{Fe}(\mathrm{II})$ binds NO forming $\mathrm{N}_{2} \mathrm{O}$ (Lo et al., 2016), which is in line with the intrinsic capacity of di-iron proteins for $\mathrm{NO}$ reduction and $\mathrm{O}_{2}$ activation/reduction (Lu et al., 2019). Moreover, in YtfE one of the iron ions of the center is loosely bound with iron dissociation constants in a range of values that enables YtfE to donate iron to other proteins (Nobre et al., 2014).

In bacteria, the biogenesis of $\mathrm{Fe}-\mathrm{S}$ clusters requires specialized machineries, ISC (iron sulfur cluster) being one of the main operative systems. As in many other bacteria, in $E$. coli the first steps of the biogenesis involve IscS, a cysteine desulfurase that catalyzes the sulfur mobilization from cysteine, an iron donor protein, and the scaffold IscU protein where the Fe-S clusters are assembled prior to their transport and insertion into the target apo-proteins (Zheng et al., 1998; Schwartz et al., 2000; Tokumoto et al., 2002; Kim et al., 2009; Roche et al., 2013; Blanc et al., 2015). We previously provided evidences that $E$. coli $\mathrm{YtfE}$ acts as an iron donor for the in vitro formation of Fe-S clusters in IscU (Nobre et al., 2014). In this work, we show that YtfE interacts directly with IscS and IscU. The analysis of the biochemical properties and crystal structures of E. coli YtfE wild-type and several sitedirected mutants allowed us to identify two glutamates, E125 and E159, with a key role in supporting the ability of YtfE to act as an iron donor protein.

\section{RESULTS}

\section{YtfE Interacts With IscS and IscU for Fe-S Cluster Biogenesis}

We previously reported that $E$. coli YtfE donates iron to form an Fe-S cluster in E. coli IscU in a in vitro reaction that also required the presence of IscS (Nobre et al., 2014). This observation led us to examine whether YtfE interacts with IscS and IscU using a genetic approach, a complementation fluorescence assay and pulldown experiments.

For the genetic approach, we measured the $\beta$-galactosidase activities of cells transformed with a pair of plasmids derived from the high-copy number vector pUT18 and the low-copy number vector pKT25 (Karimova et al., 1998). These vectors express YtfE, IscS, and IscU proteins as fusions to the $N$ - and $C$-terminal of T25 domain (NT25 and T25, respectively) and to the $N$ - and $C$-terminal of T18 domain (T18 and T18C, respectively) of the adenylate cyclase enzyme (see section "Materials and Methods"). The YtfE-YtfE interaction was used as positive control, as YtfE self-associates forming dimers (Justino et al., 2006).
We observed the formation of a complex between YtfE and IscS and IscU (Figure 1A). The $\beta$-galactosidase activity of cells containing YtfE and IscS was 2-4 times higher than that of the controls (i.e., of cells containing pUT18/18CIscS and pKT25/NT25, and of cells with pUT18/18C and pKT25/NT25-YtfE) for two of the possible protein interacting conformations. Concerning the YtfE-IscU interaction, only the conformation in which $\mathrm{Isc} U$ was expressed from the $N$-terminal part of the pUT18 fusion protein yielded significant $\beta$-galactosidase activity.

A pulldown assay was also used to show the interaction between YtfE and Isc proteins. In this method, we grew cells transformed with plasmids expressing YtfE non-tagged, IscS and $\mathrm{IscU}$ (both fused at the $\mathrm{N}$-terminal to a H6-tag) and IscU linked $C$-terminally to a S-tag, that prior to be collected were treated with $1 \%$ of formaldehyde to promote in vivo cross-linking. Cells expressing only one of the proteins were treated and analyzed similarly to serve as controls.

Cell extracts containing IscS-H6 or IscU-H6 were loaded into columns, and the Isc-H6 proteins bound strongly to the matrix, being eluted at $1 \mathrm{M}$ of imidazole (Figure 1B, lanes 2-3). On the contrary, analysis of cell extracts expressing, separately, YtfE or IscU-S, showed that these proteins do not bind to the resin (lanes Figure 1B, 1 and 4). As expected, IscS-H6 retains IscU-S due to the interaction of the two proteins (Figure 1B, lane 5), a result that is in agreement with previous studies on the IscU/IscS interaction analysed by other methods (Kato et al., 2002; Prischi et al., 2010; Kim et al., 2015).

We observed that both IscS-H6 and IscU-H6 retain the nontagged YtfE (Figure 1B, lanes 7 and 8, respectively). Furthermore, when cells express simultaneously IscS-H6, non-labeled-YtfE and IscU-S (in which the last proteins cannot bind by itself to the $\mathrm{Ni}$ resin) co-elution of the three proteins was observed (Figure 1B lane 6), further proving their interaction.

Lastly, we used Bimolecular Complementation Fluorescence (BiFC), that is based on the reconstitution of the green fluorescent protein (GFP) protein, to analyze the interaction of the proteins in vivo. GFP-fragment fusions were linked to the $N$ - and $C$-terminal domains of $\mathrm{YtfE}$, IscU, and IscS so that all possible combinations were tested. E. coli cells containing plasmids coexpressing $\mathrm{YtfE} / \mathrm{Isc} \mathrm{U}, \mathrm{YtfE} / \mathrm{IscS}$, and $\mathrm{IscS} / \mathrm{IscU}$ fusions, prepared as described in Methods, were spread onto agarose slides and the microscopy images and fluorescence intensity values are shown in Figure 1C.

As expected, cells expressing Isc $\mathrm{U}^{\mathrm{N}-\mathrm{GFP}} / \mathrm{IscS}^{\mathrm{C}-\mathrm{GFP}}$ exhibited a positive interaction with a 3 -fold higher fluorescence than the control sample. More important, the E. coli cells expressing separately $\mathrm{IscU}^{\mathrm{N}-\mathrm{GFP}} / \mathrm{YtfE}^{\mathrm{C}-\mathrm{GFP}}$ and $\mathrm{YtfE}^{\mathrm{N}-\mathrm{GFP}} / \mathrm{IscS}^{\mathrm{C}-\mathrm{GFP}}$ exhibited higher fluorescence values by approximately 3fold and 6-fold, respectively, relative to the control (empty N-GFP/C-GFP plasmids) (Figure 1C). Moreover, the interactions are conformationally dependent as cells expressing $\mathrm{YtfE}^{\mathrm{N}-\mathrm{GFP}} / \mathrm{IscU}^{\mathrm{C}-\mathrm{GFP}}$ and $\mathrm{IscS}^{\mathrm{N}-\mathrm{GFP}} / \mathrm{YtfE}^{\mathrm{C}-\mathrm{GFP}}$ had no significant fluorescence.

Altogether, the results clearly show that YtfE interacts with the two major proteins of the E. coli ISC system, namely IscS and IscU. 

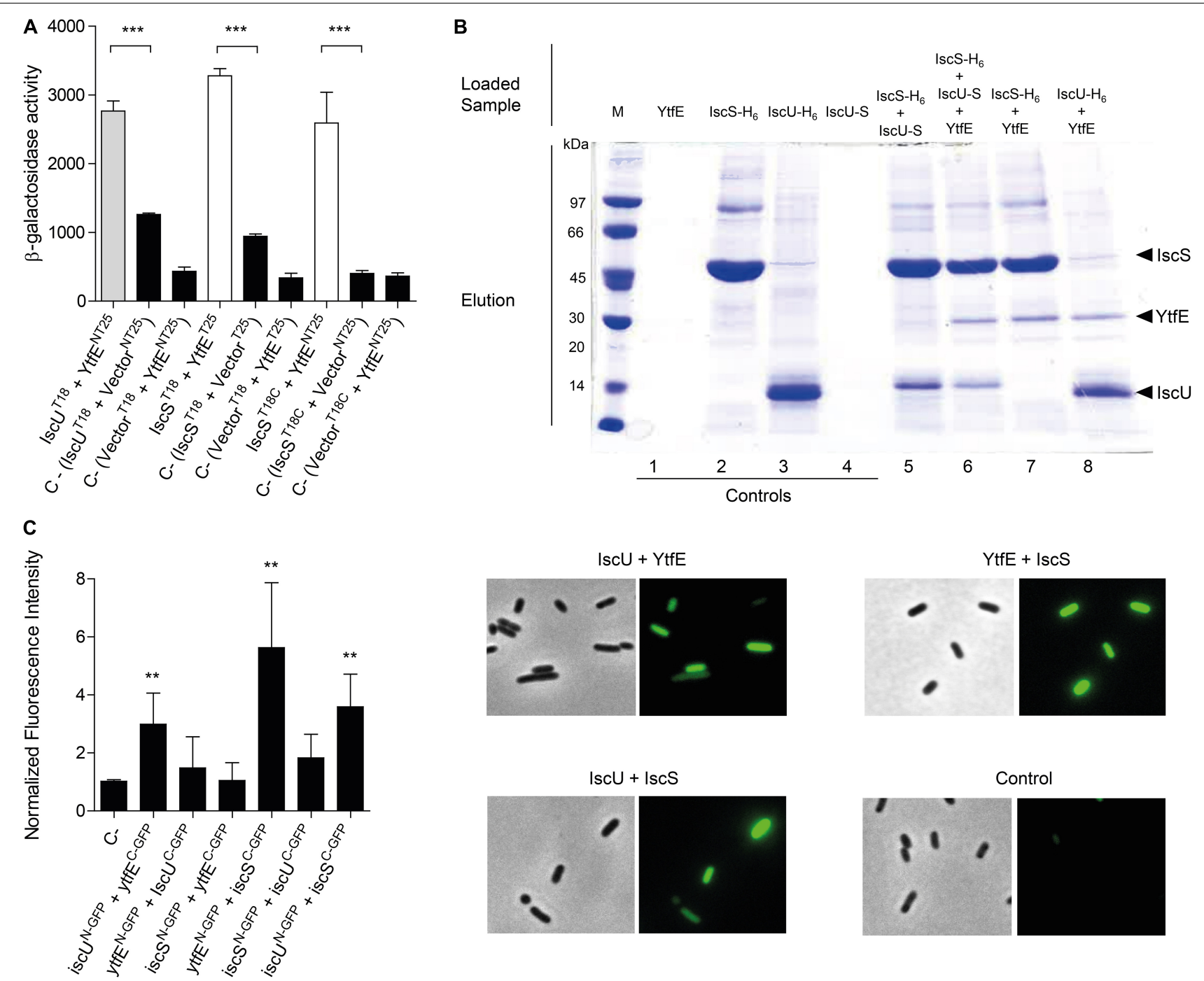

FIGURE 1 | Analysis of the interaction of YtfE with IscS and IscU. (A) The efficiency of the functional complementation between hybrid proteins was measured by $\beta$-galactosidase assays. The interaction of YtfE with IscU (gray bar) and IscS (white bars) was evaluated in E. coli cells co-transformed with plasmids containing either isc $U$ or iscS genes cloned at the $\mathrm{N}$ - or $\mathrm{C}$-terminal of the T18 fragment (T18/T18C), and the plasmid harboring the ytfE gene cloned at the $\mathrm{N}$ - or $\mathrm{C}$-terminal of the T25 fragment (NT25/T25). T25/NT25 empty plasmids together with the T18/18C plasmid containing either the isc $U$ or the iscS gene or T18/18C empty plasmid with NT25/T5 harboring ytfE were utilized as negative controls (black bars). Values are means \pm standard errors from at least three independent cultures analyzed in duplicate. ${ }^{\star \star \star} P<0.0001$; (B) For pulldown assays of YtfE, IscS and IscU, samples were obtained from cell extracts expressing one, two or three proteins previously treated with $1 \%$ formaldehyde and loaded in Ni-chelating columns, eluted with $1 \mathrm{M}$ imidazole buffer, and subjected to SDS-PAGE. Lanes 1-4: control samples from extracts expressing only one protein. Lanes 5-8: co-purification of IscS with IscU (5); co-purification of YtfE and IscU with IscS (6); co-purification of IscS with YtfE (7); and co-purification of IscU with YtfE (8); and (C) In BiCF of YtfE and ISC proteins, cells were co-transformed with vectors expressing YtfE and IscS or IscU - GFP fusions. Cells harboring empty plasmids were used as controls. On the left, fluorescence quantification was performed using MetaMorph Microscopy Automation and Image Analysis Software. Fluorescence values for the negative control (empty plasmid vectors) were normalized to $1 .{ }^{\star *} P<0.005$. On the right microscope

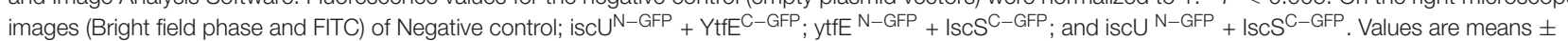
standard errors from at least three independent cultures analyzed in duplicate.

\section{E159 and E125 Modulate the Iron Release From E. coli YtfE}

Based on the data above that provides a physiological meaning to the iron donor capacity of YtfE, we sought to identify the amino acid residues that modulate these properties by using site-directed mutant proteins and determine their iron binding parameters. Our hypothesis was that these residues would be among the highly conserved amino acid residues that are located in the regions that connect the protein to the solvent. Based on the YtfE crystal structure (Lo et al., 2016), two residues could fulfill these requisites, namely E125 and E159. Thus, the wild-type protein and five YtfE proteins in which these residues were replaced by the neutral amino acid leucine and by positively or negatively charged residues, such as asparagine and aspartic 
acid, were prepared (YtfE-E125L, YtfE-E125N, YtfE-E125D, YtfEE159L, and YtfE-E159N). Furthermore, to investigate the impact on the iron releasing properties of the $\mathrm{N}$-terminal region of YtfE that is closely located to a possible second exit tunnel (Lo et al., 2016), the YtfE Truncated (lacking the first 57 residy) of the $N$-terminal) and $\mathrm{YtfE}^{\mathrm{M}}$ (a protein that contains C30AC31A double mutation used in the crystallographic study) were also studied.

Proteins exhibited an iron content of $\sim 2$ and their UV-visible spectra contained a broad band approximately at $360 \mathrm{~nm}$ (data not shown), which are features similar to those of the wild-type YtfE (Justino et al., 2006; Nobre et al., 2015). The only exception was the YtfE-E159L mutant that had a lower iron content, which is in agreement with our previous results (Nobre et al., 2015).

To determine the iron binding chemistry, the proteins were incubated with the iron chelator desferrioxamine and the formation of the desferrioxamine-Fe(III) complex was monitored by following the intensity of the $420 \mathrm{~nm}$ band. The dissociation constant $\mathrm{K}_{\mathrm{d}}$ of Ytfe-Fe(III) was calculated using the equation described in Methods. In addition, the kinetics of YtfE iron release was also determined through the initial ferric iron release rate $\left(V_{0}\right)$, which was calculated from the slope of a linear fit to the curve obtained when using the highest concentration of chelator $(1,000 \mu \mathrm{M}$; Table 1 and Supplementary Figures $\mathbf{1}, \mathbf{2})$.

Table 1 shows that the $K_{d}$ values of YtfE, $\mathrm{YtfE}^{\mathrm{M}}$, and YtfEE159N do not differ substantially, while YtfE-E159L presents a slightly lower value that indicates a more loosely bound iron. On the contrary, YtfE ${ }^{\text {Truncated }}$ and the proteins containing mutations in E125 exhibited higher $\mathrm{K}_{\mathrm{d}}$ values. In particular, the ferric dissociation constant of YtfE is substantially modified by the E125L mutation, becoming about 4-fold larger than that determined for the wild-type protein. Therefore, E125 greatly influences how strongly $\mathrm{Fe}$ (III) is bound to the YtfE scaffold.

Results in Table $\mathbf{1}$ also show that the kinetics of Fe(III) release from YtfE is controlled by E159 and E125, and is influenced by the nature of the substituent residue. Glutamate is a polar charged residue, and its replacement by the polar but uncharged residue asparagine increased the iron release rate; an even higher augment was observed (by ca. 7-fold) when E159 was substituted by the hydrophobic residue leucine.

TABLE 1 | Iron content, dissociation constants and initial iron release rates of E. coli YtfE and variants.

\begin{tabular}{lccc}
\hline Protein & Fe content* $^{*}$ & $\mathbf{K}_{\boldsymbol{d} \text { YtfE-Chelator }}\left(\mathbf{1 0}^{-\mathbf{6}} \mathbf{M}\right)$ & $\mathbf{V}_{\mathbf{0}} \mathbf{( h}^{\mathbf{- 1}} \mathbf{)}$ \\
\hline YtfE WT & $1.9 \pm 0.05$ & $46 \pm 5$ & $26 \pm 7$ \\
YtfEM $^{M}$ & $2.0 \pm 0.12$ & $46 \pm 11$ & $19 \pm 4$ \\
YtfEETruncated $^{-1.0}$ & $2.0 \pm 0.07$ & $64 \pm 9$ & $20 \pm 3$ \\
YtfE-E159N & $1.6 \pm 0.07$ & $48 \pm 8$ & $42 \pm 4$ \\
YtfE-E159L & $0.7 \pm 0.28$ & $27 \pm 9$ & $191 \pm 29$ \\
YtfE-E125N & $2.0 \pm 0.35$ & $71 \pm 16$ & $8 \pm 2$ \\
YtfE-E125D & $1.8 \pm 0.02$ & $68 \pm 22$ & $22 \pm 2$ \\
YtfE-E125L & $1.7 \pm 0.14$ & $194 \pm 52$ & $3 \pm 1$ \\
\hline
\end{tabular}

*No. of Fe atoms per monomer. $K_{d}$ and $V_{0}$ were calculated using GraphPad Prism considering at least two independent sample measurments.
Interestingly, an opposite effect occurred upon substitution of E125, originating proteins with slower iron release rates. Mutation of E125 to asparagine decreased the $\mathrm{V}_{0}$ values by about 3 -fold whereas a leucine substitution reduced the iron-release rate by $c a$. 8 -fold.

Altogether, these results revealed that glutamate E125 has a key role in the modulation of the thermodynamic and kinetic properties of the iron release from the YtfE di-iron center.

\section{E125 Is Essential for YtfE to Act as Iron Donor}

Considering the results described above, we next determined the impact of the E125 mutation on the YtfE iron donor capacity to assist formation of a Fe-S center in the ISC system. With this in mind, reactions mixtures containing E. coli apoIscU, YtfE (wild-type or mutant proteins), IscS, L-cysteine and DTT (1,4-dithiothreitol), were monitored under anaerobic conditions and the cluster formation in $\mathrm{IscU}$ was monitored by visible spectroscopy.

The spectrum of the reaction mixture containing Isc $\mathrm{U}$ and wild-type YtfE, obtained after a period of incubation of $1 \mathrm{~h}$, exhibited bands at $\sim 456 \mathrm{~nm}$ and $\sim 410 \mathrm{~nm}$ characteristic of the presence of a mixture of $[2 \mathrm{Fe}-2 \mathrm{~S}]^{2+/ 1+}$ and $[4 \mathrm{Fe}-4 \mathrm{~S}]^{2+/ 1+}$ clusters (Supplementary Figure 3). On the contrary, the spectra of the reaction mixtures containing IscU and YtfE-E125N or IscU and YtfE-E125L exhibited very weak bands (Supplementary Figure 3), which indicates that the amount of ISC formed in $\mathrm{Isc} \mathrm{U}$ is negligible.

These results are consistent with the thermodynamic and kinetic properties of YtfE-E125N and YtfE-E125L which show that in these proteins the iron is more tightly bound and the rate of iron release is much lower.

Hence, the mutation of E125 by a hydrophobic or a positively charged residue hindered the iron donation capacity of YtfE which led us to conclude that residue E125 plays a key role on the protein function.

\section{Structural Basis for the Iron Donor Properties of YtfE}

The unexpected iron binding properties of the YtfE-E159 and YtfE-E125 mutant proteins, in which the modified amino acid residues are located outside of the coordination sphere of the di-iron center (Figure 2A), prompted us to address the structural role of two glutamates in YtfE. We resorted to $\mathrm{X}$-ray crystallography to determine their structures and, for comparison purposes, we revisited the structure of the wildtype YtfE.

Crystals of E. coli YtfE could only be obtained when the proteins contained the mutation to alanine of the two vicinal cysteines C30 and C31 (designated $\mathrm{YtfE}^{\mathrm{M}}$ ), a result that agrees with previous reports (Lo et al., 2016). Therefore, we prepared crystals of $\mathrm{YtfE}^{\mathrm{M}}$, $\mathrm{YtfE}^{\mathrm{M}}-\mathrm{E} 159 \mathrm{~L}$, and $\mathrm{YtfE}^{\mathrm{M}}-\mathrm{E} 125 \mathrm{~L}$ as described in Methods. The atomic coordinates and experimental structure factors were deposited in the Worldwide Protein Data Bank (Burley et al., 2019) with the accession codes 7BHA, 7BHB, and 

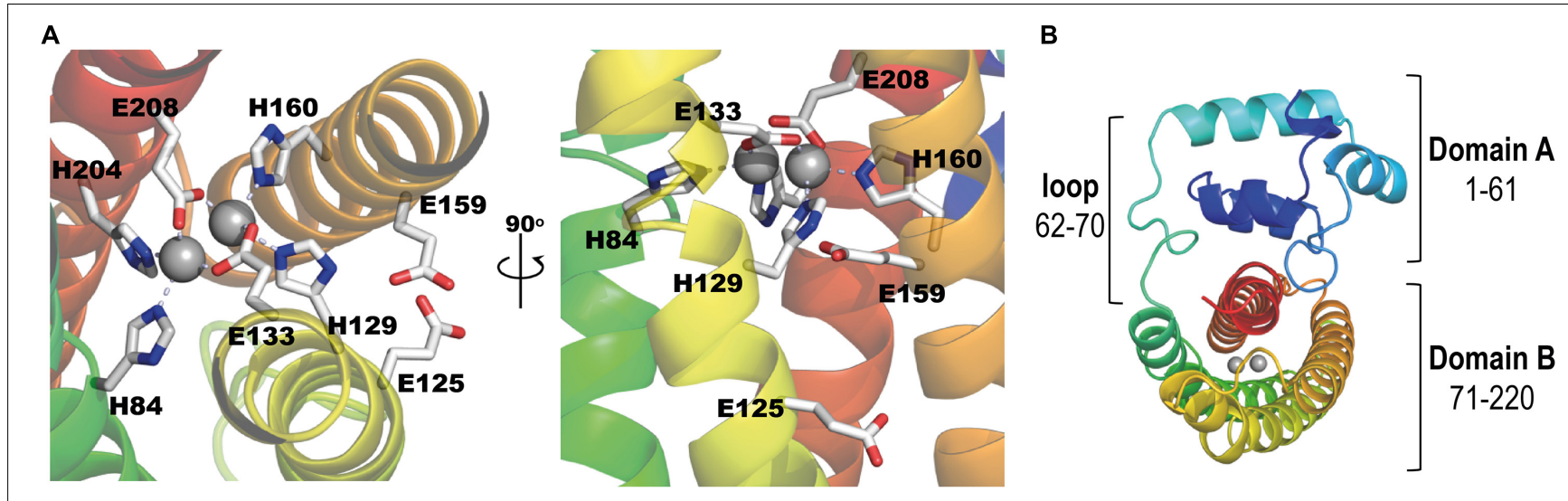

C

YtfEM

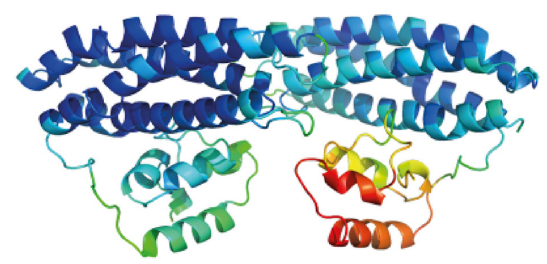

YtfEM-E159L

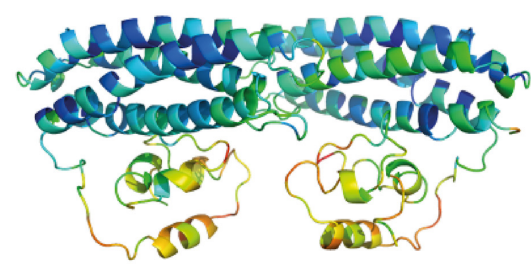

YtfEM-E125L

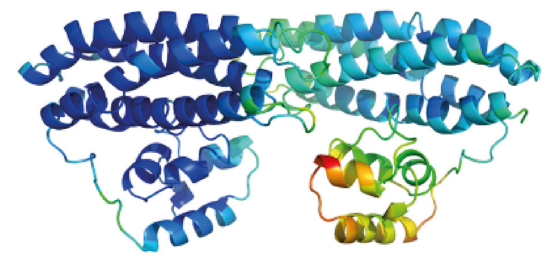

FIGURE 2 | E. coli YtfE ${ }^{\mathrm{M}}$ overall structure. (A) Detail views of the YtfE ${ }^{\mathrm{M}}$ di-iron center, its coordinating residues, and residues E125 and E159 highlighted in light gray (left panel view is rotated $90^{\circ}$ about a vertical axis from first panel). (B) Cartoon representation of the E. coli YtfE $^{\mathrm{M}}$ monomer (chain A) showing the two domains that are connected by a loop. In (A,B) the monomer structure is rainbow colored from dark blue in the $\mathrm{N}$-terminal to red in the $\mathrm{C}$-terminal. The di-iron site is represented as gray spheres. (C) Cartoon representation of the $E$. coli $\mathrm{YtfE}^{\mathrm{M}}$, $\mathrm{YtfE}^{\mathrm{M}}-\mathrm{E} 159 \mathrm{~L}$, and $\mathrm{YtfE}^{\mathrm{M}}-\mathrm{E} 125 \mathrm{~L}$ subunits present in the asymmetric unit, colored according to their B-factor values (the di-iron site is omitted).

7BHC for the $\mathrm{YtfE}^{\mathrm{M}}, \mathrm{YtfE}^{\mathrm{M}}-\mathrm{E} 159 \mathrm{~L}$, and $\mathrm{YtfE}^{\mathrm{M}}-\mathrm{E} 125 \mathrm{~L}$ structures, respectively. Data collection and refinement statistics are listed in Supplementary Tables 1, 2.

The three crystal structures contain two molecules in the asymmetric unit (chains $\mathrm{A}$ and $\mathrm{B}$ ) in monoclinic space group $P 2_{1}$. All molecules are composed of two domains (A and $\mathrm{B}$ ) and have continuous electron density for the full length of the protein (220 residues; Figure 2B). However, in all the structures here presented, the electron density for chain $B$ is overall weaker than for chain $A$, as inferred from their mean $B$-values (see Supplementary Table 3), and therefore only chain $A$ will be considered for the following structural analysis. The crystal structure of $\mathrm{YtfE}^{\mathrm{M}}$ $\mathrm{E} 125 \mathrm{~L}$ resembles more that of $\mathrm{YtfE}^{\mathrm{M}}$. However, in $\mathrm{YtfE}^{\mathrm{M}}$ E159L more regions with low density are observed, and the highest $B$-values are associated with domain A (Figure $2 \mathrm{C}$ and Supplementary Table 3).

Domain A, usually referred to as a ScdA_N-like domain (Brunskill et al., 1997; Chang et al., 2006) is composed of $4 \alpha$-helices, and contains the DxCCG motif in the loop between helices 2 and 3, which is highly conserved in the RIC family (Supplementary Figure 4). The structures exhibited a domain A fold similar to the previous reported YtfE wild-type structure (Lo et al., 2016) with a r.m.s.d. (root-mean-square deviation) of only $0.3 \AA$ between $188-193$ superposed main-chain $\mathrm{C} \alpha$ atoms.
In the three structures, several residues in domain $\mathrm{A}$ can establish intermolecular contacts with symmetry-related monomers. In $\mathrm{YtfE}^{\mathrm{M}}$, hydrogen bonds occur between residues D5 and R17, E49, and R86. In the $\mathrm{YtfE}^{\mathrm{M}}-\mathrm{E} 125 \mathrm{~L}$ structure, the same hydrogen bonds are present together with bonds between R39 and K57/E60/Q61. Interestingly, in the $\mathrm{YtfE}^{\mathrm{M}}$-E159L structure, no such hydrogen bond interactions are present, probably explaining why the $B$-values in this domain are higher than in the other structures (Figure 2C and Supplementary Table 3).

Like for domain A, the overall four-helix bundle structure of domain $\mathrm{B}$ is maintained independently of the mutation introduced in the protein. As noted earlier (Todorovic et al., 2008; Nobre et al., 2015; Alvarez-Carreño et al., 2018), domain B shares a similar structural topology with proteins such as hemerythrins, rubrerythrins, and (bacterio)ferritins, with a r.m.s.d. of ca. $3 \AA$ between superposed main-chain $\mathrm{C} \alpha$ atoms (Supplementary Figure 5). An extensive structural analysis of the available hemerythrin-like proteins (Alvarez-Carreño et al., 2018), rubrerythrins, and (bacterio)ferritins, revealed that the arrangement of the helices in YtfE is unique in its lefthanded four-helix bundle structure. Hemerythrins have righthanded four-helix bundles (Supplementary Figure 5b), and rubrerythrins or (bacterio)ferritins exhibit a mixed arrangement, starting as left-handed and then becoming a right-handed fourhelix bundle (Supplementary Figures 5c,d). These results show that in relation to the structural fold of the four-helix bundle, YtfE 


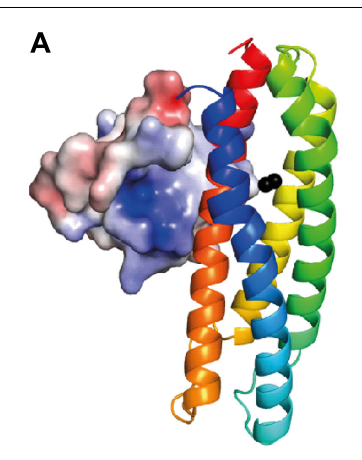

B

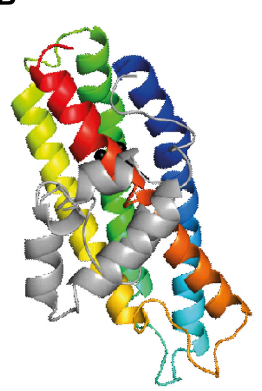

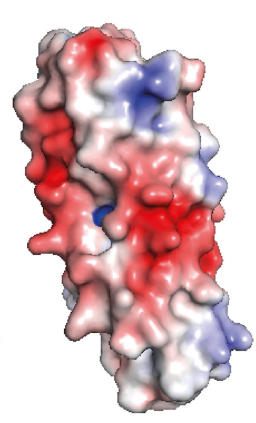

C

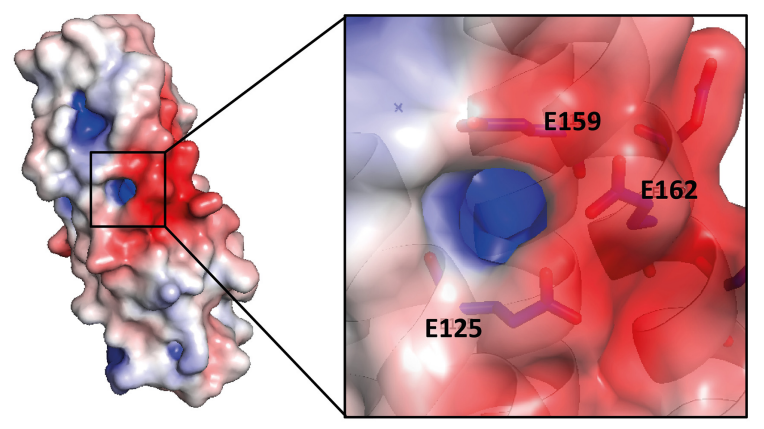

$=5$

\section{$k T / e$}

FIGURE 3 | Electrostatic potential at the molecular surface of E. coli YtfEM . (A) Electrostatic potential surface of domain A that faces domain B is positively charged. (B) View of $\mathrm{YtfE}^{\mathrm{M}}$ rotated $90^{\circ}$ about a vertical axis from panel $\mathbf{A}$, the electrostatic surface representation (right) is on the same orientation as the cartoon representation of the structure (left), without domain A. (C) Detail of the electrostatic surface (negatively charged) near the iron cavity (positively charged). The electrostatic potential at the molecular surface was obtained considering that $\mathrm{YtfE}^{\mathrm{M}}$ is loaded with $\mathrm{Fe}^{3+} \mathrm{Fe}^{2+}$. The electrostatic potential surfaces range from -5 kT/e (red) to +5 kT/e (blue). Figure prepared with PyMOL (DeLano, 2020) using ABPS (Baker et al., 2001) and PDB2PQR (Dolinsky et al., 2004) server to generate the electrostatic potential surface. The charge for each metal was manually added to the PQR file, taking in consideration the oxidation state of the protein.

and RIC proteins form a separate cluster within the hemerythrin and ferritin-like families.

Analysis of the electrostatic potential on the molecular surface of E. coli $\mathrm{YtfE}^{\mathrm{M}}$ shows that domain A is mainly positively charged and domain B is mainly negatively charged (Figures $\mathbf{3 A}, \mathbf{B}$ ). In addition, the cavity where the di-iron center is inserted is positively charged, and the negative charge at the surface in this region is mainly due to residues E125, E159, and E162 (Figure 3C).

\section{Structural Features of the Di-Metal Site}

Escherichia coli $\mathrm{YtfE}^{\mathrm{M}}$ and $\mathrm{YtfE}^{\mathrm{M}}$-E125L contain a di-iron site coordinated by four histidine residues, through their $\mathrm{N}^{\varepsilon 2}$ atoms (H84 and $\mathrm{H} 204$ for Fe1, H129 and $\mathrm{H} 160$ for $\mathrm{Fe} 2$ ), and two bidentate glutamic acids that bridge the two irons (E133, E208; Figures 4A,C). These residues are part of the H84 $\left(\mathrm{x}_{44}\right) \mathrm{H} 129\left(\mathrm{x}_{3}\right) \mathrm{E} 133\left(\mathrm{x}_{26}\right) \mathrm{H} 160\left(\mathrm{x}_{43}\right) \mathrm{H} 204\left(\mathrm{x}_{3}\right) \mathrm{E} 208$ binding motif, that shares similarities with the four-histidine binding motif in hemerythins. However, in YtfE the two bridging ligands are glutamates, whereas in hemerythrins one residue is a glutamate and the other is an aspartate (Alvarez-Carreño et al., 2018). Besides these coordinating ligands, an oxo-bridge between the iron atoms is also present in YtfE. Except for $\mathrm{YtfE}^{\mathrm{M}}$-E159L that only contains one iron atom in the center, the di-iron coordination and the distances of the ligands to the irons are similar in the $\mathrm{YtfE}^{\mathrm{M}}$ and $\mathrm{YtfE}^{\mathrm{M}}-\mathrm{E} 125 \mathrm{~L}$ structures. Supplementary Table 4 lists details of the coordinating distances of the structures.

As mentioned above, $\mathrm{YtfE}^{\mathrm{M}}$-E159L contains only one iron atom in the metal site (Figure 4B and Supplementary Figure 6). Additionally, the conformation of ligand H129 differs from that observed in the other two proteins. Specifically, the side chain of H129 is $\sim 7 \AA$ apart from its original position and no longer pointing toward the di-iron center (the closest distance to $\mathrm{YtfE}^{\mathrm{M}}$ E159L H129 is ca. $9 \AA$ whereas in $\mathrm{Ytfe}^{\mathrm{M}}$ it is about $2 \AA$ ).

\section{The Iron Exit Channel Is Controlled by E125}

The structure of E. coli $\mathrm{YtfE}^{\mathrm{M}}$ contains two channels (Lo et al., 2016). One channel is mainly hydrophobic (which will not be addressed in this study), and a second hydrophilic channel that connects the metal center to the solvent (Figure 5A). The diiron center is located $c a .10 \AA$ below the channel entrance, which is approximately circular in shape with a diameter of $\sim 2.3 \AA$. The channel is formed mainly by hydrophilic residues, namely H160 and H129 (that are also Fe ligands), K132, E159, E125, and E162. The three negatively charged glutamates are located at the channel entrance and solvent exposed suggesting a possible iron exit (Lo et al., 2016). Hence, mutation of these residues was done and the channel in $\mathrm{YtfE}^{\mathrm{M}}$ was compared with that present in the $\mathrm{YtfE}^{\mathrm{M}}$-E159L and $\mathrm{YtfE}^{\mathrm{M}}$-E125L mutant proteins.

The structure of $\mathrm{YtfE}^{\mathrm{M}}$-E159L contains only one iron atom in the metal center that is located $c a$. $13 \AA$ below the channel entrance. In this case, the channel is longer, with a length of about $16 \AA$, and although the channel entrance retains the circular shape, its diameter is reduced to $\sim 1.5 \AA$ (Figure 5B). The channel is also formed by hydrophilic residues, namely by the ligands H204, H129, H160, and residues E125, E162, and L166 located at the channel entrance. In $\mathrm{YtfE}^{\mathrm{M}}-\mathrm{E} 159 \mathrm{~L}$, the absence of the second Fe atom, the different position of the H129 sidechain (mentioned above) and the presence of leucine in position 159 modified the channel arrangement so that the substituting L159 residue is no longer included in the channel (Figures 5A,B and Supplementary Figure 6).

The mutation in $\mathrm{YtfE}^{\mathrm{M}}$-E125L induced a major modification of the channel (Figure 5C). In this case, a significant shortening 

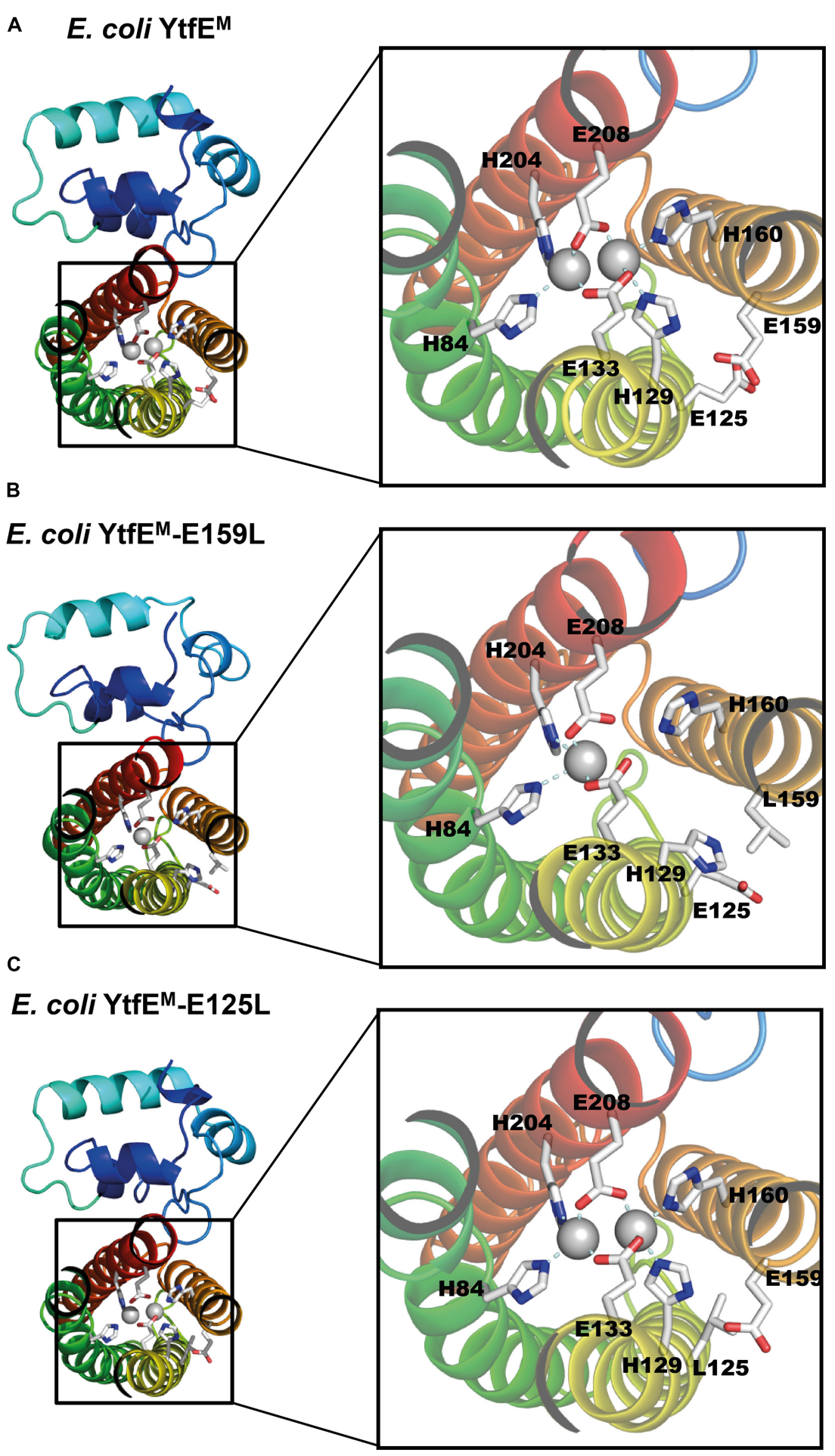

FIGURE 4 | E. coli YtfE Iron center. (A) YtfE ${ }^{\mathrm{M}}$ di-iron center is coordinated by residues H84, H129, E133, H160, H204, and E208. (B) YtfEM -E159L mononuclear Fe center is coordinated by $\mathrm{H} 84, \mathrm{H} 2 \mathrm{O} 4$ and E208. The second Fe atom is not present and residue $\mathrm{H} 129$ assumes a different side chain conformation from that in YtfEM (C) In YtfEM-E125L the di-iron center, is structurally similar to in YttEM .

of the distance between the sidechains of residues L125 and E159 is observed, from $8.5 \AA$ in $\mathrm{YtfE}^{\mathrm{M}}$ to $5.3 \AA$ in $\mathrm{YtfE}^{\mathrm{M}}-\mathrm{E} 125 \mathrm{~L}$ (Figure 5D). As a consequence, the new position of L125 results in the occlusion of the channel and, consequently, the di-iron center is no longer exposed to the solvent. The closing of the channel is consistent with the different iron release properties 


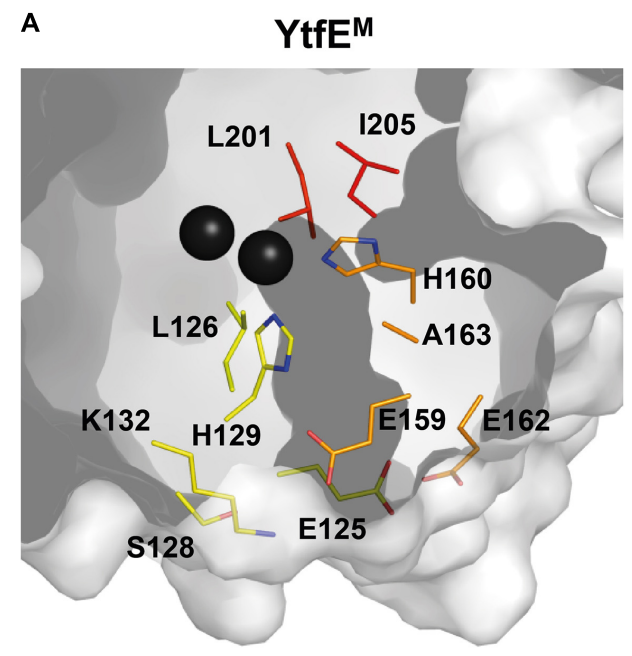

c

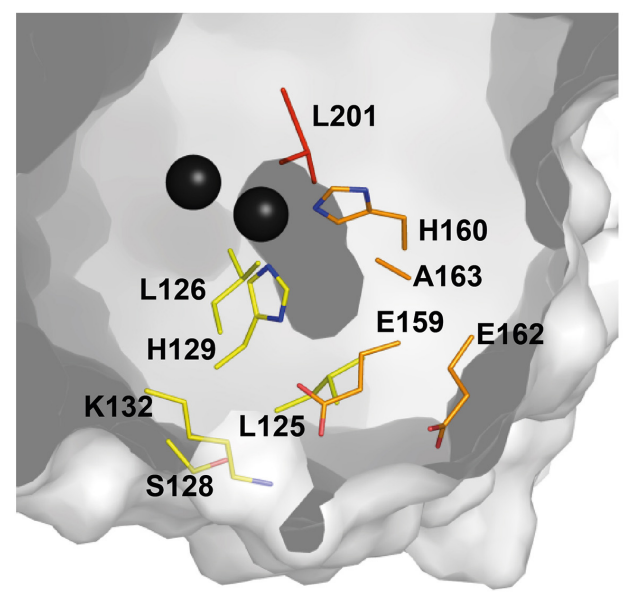

B

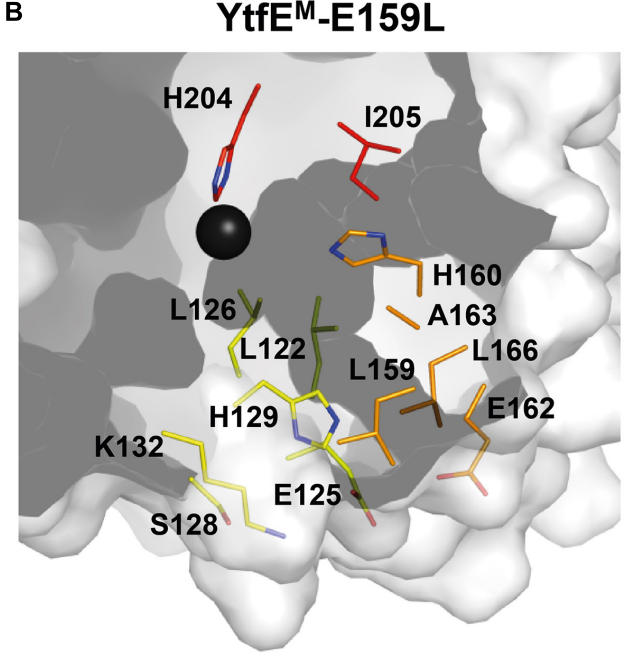

D

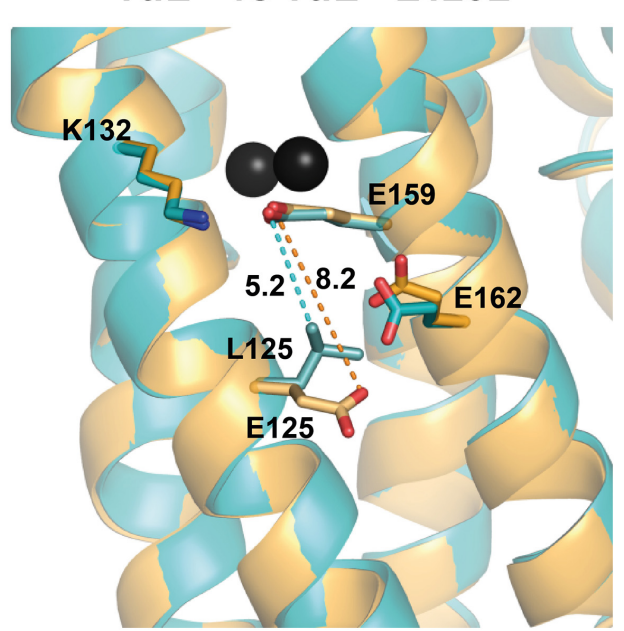

FIGURE 5 | Channels present in $\mathrm{YtFE}^{\mathrm{M}}$ and $\mathrm{YttE}^{\mathrm{M}}$-E159L structures. Channels are represented in dark gray. Residues lining the iron channel are represented as sticks with carbon atoms rainbow colored according to the helices (helix 2 - yellow, helix 3 - orange, and helix 4 - red). (A) YttE ${ }^{\mathrm{M}}$ iron channel connects the external surface to the di-iron site. YttE ${ }^{\mathrm{M}}$ has its channel entrance formed by residues E159, E162, E125, and K132. (B) In YttE ${ }^{\mathrm{M}}$-E159L, the channel connects the external surface to the mononuclear iron center, and has its entrance between residues E162, L166, and E125, i.e., slightly below that of the YtfEM pore. (C) No channels are observed in the YtfE ${ }^{\mathrm{M}}$-E125L structure. A small cavity occurs near the di-iron center, however, the channel is interrupted by mutation of E125 to leucine.

(D) Superposition of the $\mathrm{YtfE}^{\mathrm{M}}$ (orange) and $\mathrm{YtfE}^{\mathrm{M}}$-E125 (cyan) structures showing the distances between the residues involved in the channel entrance. Distance between YtfEM residues 159 and 125 is represented by a dashed orange line whereas the distance between $\mathrm{YtfE}^{\mathrm{M}}$-E125L residues is indicated by a dashed cyan line. The channels were modeled using MOLE (Sehnal et al., 2013) and PyMOL (DeLano, 2020) software.

of the $\mathrm{YtfE}^{\mathrm{M}}-\mathrm{E} 125 \mathrm{~L}$, and explains why this mutant protein is no longer able to promote the formation of the Fe-S center in IscS, as described above.

\section{DISCUSSION}

Previously, we showed that E. coli YtfE is a major candidate to be an iron donor for IscU, based on two experimental observations. First, the YtfE dissociation constant for iron is lower than those of other potential iron donors, namely CyaY, YggX, IscA, and SufA (Ding and Clark, 2004; Skovran et al., 2004; Layer et al., 2006; Lu et al., 2008; Nobre et al., 2014). Second, YtfE can provide iron to
$\mathrm{IscU}$ in $\mathrm{Fe}-\mathrm{S}$ cluster reconstitution assays in vitro via a cysteinemediated process. In this work, we proved that YtfE interacts with IscU and IscS, and revealed the structural features that underpin the YtfE iron donation properties.

We re-analyzed the wild-type $\mathrm{YtfE}^{\mathrm{M}}$ and determined the structures of $\mathrm{YtfE}^{\mathrm{M}}$-E125L and $\mathrm{YtfE}^{\mathrm{M}}$-E159L mutants. In contrast with the previously reported $E$. coli YtfE structure $(5 \mathrm{FNN}$; Lo et al., 2016), the structure herein presented shows a full occupancy of the di-iron site. Moreover, residues that were not visible in the $5 \mathrm{FNN}$ structure are now modeled, and the loop comprising residues 54-65 in chain B exhibits several differences. Although the mutations did not change the overall structure, the single replacement by uncharged amino acids of 
the negatively charged amino acid residues E159 and E125, which are not ligands of the di-iron center, introduced significant changes locally which were sufficient to impair the function of the protein. In E. coli YtfE, the highly conserved residue E159 is located at about $7 \AA$ from the closest iron atom, therefore it is not involved in metal coordination. Replacement of E159 with another polar amino acid, YtfE-E159N, generated a protein with $\mathrm{K}_{\mathrm{d}}$ values similar to those of the wild-type. However, in YtfEE159L with a neutral leucine instead of the glutamate, the metal site lost one of its iron ions. Thermodynamic and kinetic data indicate that mutation of E159 generates a protein with a more unstable center that releases iron at a higher rate. Moreover, the structure of $\mathrm{YtfE}^{\mathrm{M}}$ revealed that when E159, that establishes a hydrogen bond with the iron ligand $\mathrm{H} 129\left(\mathrm{H}_{12} 9^{N \delta 1} \cdot \mathrm{E} 159^{\mathrm{O} \varepsilon 1}\right.$ $2.66 \AA$ ), is replaced by leucine, the position of the neighboring histidine H129 alters its sidechain conformation, pointing away from the center and toward the protein surface (Figure 4B and Supplementary Figure 6). Thus, the intermolecular interaction between H129 and E159 is crucial for the overall stability of the center. It is also interesting to note that in our previous work, H129 appeared to be the only histidine ligand that controls the ability of YtfE to donate iron (Nobre et al., 2015).

The mutation of E125 (which is located at ca. $9 \AA$ from its nearest iron in the di-iron center) to leucine generated a protein with an intact binuclear site. However, and unexpectedly, the YtfE-E125L mutant has a hindered capacity to release iron and is not able to promote the assembly of the iron-sulfur center in IscU. These results were rationalized by the analysis of the crystal structure of $\mathrm{YtfE}^{\mathrm{M}}-\mathrm{E} 125 \mathrm{~L}$ showing that the positioning of the L125 sidechain blocks the external part of the hydrophilic channel, and consequently impairs the release of iron from the di-iron cluster to protein exterior.

Thus, we have identified a mechanism through which a di-iron protein has the possibility of releasing iron as long as it contains in the upper part a set of negatively charged glutamate residues forming a channel that links the di-iron center to the surface. The mononuclear iron YtfE-E159L mimics an YtfE protein following iron release and its structure shows that the process implies the movement of one of the axial histidines that exposes the metal site cavity to the exterior, thus making possible the re-entry of another iron atom to rebuild the di-iron center. Since we previously showed that YtfE interacts with E. coli Dps (DNA-binding Protein from Starved cells; Silva L.S.O. et al., 2018), we may hypothesize that this iron storage protein constitutes a good candidate to promote the reconstitution of the di-iron cluster in YtfE.

An identical mechanism is proposed to occur in the proteins of the RIC family, as residues located equivalently to H129, E125, and E159 are conserved in the vast majority of RICs. Furthermore, our results open the possibility that this a common dynamic behavior of di-iron proteins that share similar structural features, such as bacterioferritins (Tatur et al., 2007). For example, when Desulfovibrio desulfuricans bacterioferritin is fully reduced and subsequently allowed to oxidize in the presence of atmospheric oxygen, it loses one of the iron atoms of the ferroxidase center (Macedo et al., 2003) through a not yet identified mechanism. Like RIC proteins, (bacterio) ferritins also contain a pore 6 to $7 \AA$ deep that connects directly the di-iron ferroxidase center to the surface, that has been proposed to be involved in the entry of ferrous iron to the center (Macedo et al., 2003; Swartz et al., 2006; Weeratunga et al., 2010; Rui et al., 2012; Ebrahimi et al., 2015). Moreover, the movement of histidine and glutamic acid residues is also observed and predicted to be related to the opening of the ferroxidase center to the solvent (Macedo et al., 2003; Swartz et al., 2006; Weeratunga et al., 2010). Our observations for YtfE could explain how bacterioferritins might donate iron from their di-iron center.

Despite the importance in all organisms of the Fe-S assembly systems and, in particular, of the ISC system operative in bacteria, the nature of the iron donor has remained elusive. Although frataxin (CyaY) was initially proposed to function as an iron storage/donor for $\mathrm{Isc} U$, the most recent studies indicate that frataxin is mainly an accelerator of persufilde transfer (Roche et al., 2015). Other candidates for iron donors belong to the A-type protein family that includes IscA, NifA, and SufA from various organisms, which were shown to bind ferric iron with high affinity and to provide iron to IscU. However, the IscA proteins interact only with the late-acting components of the $\mathrm{Fe}$ $S$ cluster biogenesis pathway, which has reinforced the idea that they are not involved in iron insertion (Tan et al., 2009; Vinella et al., 2009). In this work, we prove that YtfE interacts with IscU and IscS, and we have identified in the structure of YtfE a channel through which the iron may exit to promote the formation of the cluster assisted by Isc proteins. Altogether, the results now presented strongly support the proposal that in a large number of bacteria the di-iron RICs act as iron donors for the biogenesis of Fe-S clusters.

\section{MATERIALS AND METHODS}

\section{Protein-Protein Interaction Experiments Bacterial Two-Hybrid System}

The system based on the interaction-mediated reconstitution of the Bordetella pertussis adenylate cyclase (Cya) activity in E. coli (Karimova et al., 1998) was used to analyze the interaction of YtfE with IscU and IscS proteins.

The genes coding for IscS, IscU, and YtfE were PCR-amplified using appropriate pairs of oligonucleotides (Supplementary Table 5) and genomic DNA from E. coli $\mathrm{K} 12$ as templates. The generated DNA fragments were digested with BamHI and $K p n \mathrm{I}$ (for iscS and iscU genes) or with BamHI and SalI (for $y t f E$ gene), and cloned into the corresponding sites of pKT25, pKNT25, pUT18, and pUT18C (Supplementary Table 6). The recombinant plasmids allowed the production of proteins linked to the $C$ - and $N$-termini of the T25 domain and to the $N$ and C-termini of the T18 domain of B. pertussis Cya. All recombinant clones were sequenced confirming the absence of undesired mutations. The complementary plasmids (carrying a T25 fragment and a T18 fragment in all possible combinations) were co-transformed into E. coli DHM1, a strain deleted in the cya gene. The pUT18/18C or pKT25/NT25 empty plasmids cotransformed with the complementary plasmids containing iscS, $i s c U$, or $y t f E$ were used as negative controls. 
The efficiency of the interactions between the hybrid proteins was quantified by the $\beta$-galactosidase activity method using $E$. coli DHM1 as the recipient strain. At least 3-4 representative colonies of each transformation plate were inoculated, in duplicate, in $\mathrm{LB}$ broth and, following an overnight growth at $37^{\circ} \mathrm{C}$, cells were re-inoculated $1 / 100$ in LB containing ampicillin, kanamycin and isopropyl-1-thio- $\beta$-D-galactopyranoside (IPTG). When cells reached an $\mathrm{OD}_{600} \sim 0.5$ (approximately after $16 \mathrm{~h}$ growth at $\left.30^{\circ} \mathrm{C}\right), 1 \mathrm{~mL}$ of each culture was collected by centrifugation (5 min, 5,000 rpm). The pellets were suspended in $100 \mu \mathrm{L}$ BugBuster HT $1 \mathrm{x}$ (Novagen) for cellular lysis and incubated at $37^{\circ} \mathrm{C}$ for $30 \mathrm{~min}$. Cellular debris were removed by centrifugation and each suspension $(20 \mu \mathrm{L})$ was tested in duplicate for the enzymatic reaction in a microtiter plate reader. The $\beta$-galactosidase assays were initiated upon addition of the following mixture: $0.27 \% \quad \beta$-mercaptoethanol (v/v), $0.9 \mathrm{mg} / \mathrm{mL}$ ONPG (o-nitrophenyl- $\beta$-D-galactopyranoside) in buffer $\mathrm{Z}\left(60 \mathrm{mM} \mathrm{Na} \mathrm{HPO}_{4}, 40 \mathrm{mM} \mathrm{NaH} \mathrm{PO}_{4}, 1 \mathrm{mM}\right.$ $\mathrm{MgSO}_{4}$, and $10 \mathrm{mM} \mathrm{KCl}$ ). The absorbance was measured at $420 \mathrm{~nm}$ every $2 \mathrm{~min}$, and the reaction continued, at $28^{\circ} \mathrm{C}$, for $1.5 \mathrm{~h}$. The $\beta$-galactosidase specific activity is defined in units per milligram of protein. For the conversion of microplate reader $\mathrm{Abs}_{420}$ values into standard spectrophotometer values, a correction factor of 2.2 was determined using serial dilutions of an O-nitrophenyl (ONP) solution. Positive interactions were considered for $\beta$-galactosidase activities at least two times higher relative to the negative control.

\section{Pulldown Assays}

The genes encoding YtfE, IscS, and IscU were amplified from E. coli K-12 genomic DNA by PCR using specific oligonucleotides, cloned into pFLAG-CTC (Sigma), and in pACYCDuet-1 (Novagen) that allows co-expression of two target genes fused with S-tag (S) and His 6 -tag (H6) sequences (Supplementary Tables 5, 6). Sequencing of the recombinant plasmids confirmed their integrity and the absence of undesired modifications. E. coli BL21(DE3) Gold (Agilent) was transformed with one or two plasmids: (i) pFLAG-ytfE (expressing YtfE); (ii) pACYC-IscS-H6 (expressing IscS fused to a N-terminal H6-tag); (iii) pACYC-IscU-H6 (expressing IscU fused to a $N$-terminal H6tag); (iv) pACYC-IscU-S (expressing IscU fused to a $C$-terminal S-tag); (v) pACYC-IscS-H6-IscU-S (expressing IscS with a H6-tag $N$-terminal and IscU with a $C$-terminal S-tag); (vi) pFLAGytfE and pACYC-IscS-H6; (vii) pFLAG-ytfE and pACYC-IscUH6; and (vii) pFLAG-ytfE and pACYC-IscS-H6-IscU-S. Cells harboring single recombinant plasmids (from i to iv) were used as control samples. Growth was done at $30^{\circ} \mathrm{C}$ in Terrific Broth (TB) medium supplemented with antibiotics and $100 \mu \mathrm{g} / \mathrm{mL}$ of $\mathrm{Fe}$ citrate. When the culture reached an $\mathrm{OD}_{600}$ of $0.3-$ $0.4,0.3 \mathrm{mM}$ IPTG was added to induce the expression of the $\mathrm{YtfE}$, IscS, and IscU proteins. After $5 \mathrm{~h}$, the cross-linking agent formaldehyde ( $1 \%$ final concentration) was added. Incubation proceeded at $37^{\circ} \mathrm{C}$ for $20 \mathrm{~min}$, and the reaction was stopped by incubation with $0.5 \mathrm{M}$ glycine at room temperature for $5 \mathrm{~min}$ (Silva L.S.O. et al., 2018). Cells were harvested by centrifugation, washed twice, resuspended in phosphate-buffered saline (PBS), disrupted in a French press (Thermo Scientific), and debris removed by centrifugation $(15,000 \mathrm{rpm}$ for $30 \mathrm{~min})$. The total protein concentration of the supernatants was determined by the Pierce bicinchoninic acid protein assay (Thermo Scientific). For the pulldown experiments, the supernatants (Supplementary Figure 7) were loaded into Ni-chelating Sepharose Fast Flow columns (GE Healthcare), which were previously equilibrated with $20 \mathrm{mM}$ Tris- $\mathrm{HCl}$ (pH 7.9) supplemented with $0.5 \mathrm{M} \mathrm{NaCl}$ (buffer A) containing $5 \mathrm{mM}$ imidazole. Columns were extensively washed with buffer $\mathrm{A}$, then with $60 \mathrm{mM}$ imidazole buffer, and the proteins were eluted in the buffer with $1 \mathrm{M}$ imidazole (Kato et al., 2002). The protein fractions were analyzed by SDSPAGE (12.5\%).

\section{Bimolecular Complementation Fluorescence Assays}

Bimolecular complementation fluorescence assays were done essentially as previously described (Wilson et al., 2004; Silva L.S.O. et al., 2018). Briefly, genes encoding YtfE, IscS, and IscU were PCR-amplified from genomic DNA of E. coli K-12 using the oligonucleotides described in Supplementary Table 5. DNA fragments were cloned into vectors pET11a-link-N-GFP (using XhoI/BamHI sites) and pMRBAD-link-C-GFP (using NcoI/AatII sites; Wilson et al., 2004), to construct GFP fusion proteins located at the $\mathrm{N}$ - or $\mathrm{C}$-termini, respectively. All recombinant plasmids were sequenced confirming the integrity of the genes and the absence of undesired mutations. E. coli BL21(DE3) Gold cells (Agilent) were co-transformed with recombinant pET11a-N-GFP and pMRBAD-C-GFP vectors so that several combinations were tested, namely YtfE/IscS, YtfE/IscU, and IscS/IscU. Cells co-transformed with the empty vectors pET11alink-N-GFP and pMRBAD-link-C-GFP were used as negative controls. Colonies were inoculated in LB medium, grown overnight at $37^{\circ} \mathrm{C}$ and $150 \mathrm{rpm}$, and plated on inducing and selective LB agar medium, which contained $20 \mu \mathrm{M}$ IPTG, $0.2 \%$ of arabinose and adequate antibiotics. The plates were incubated overnight at $30^{\circ} \mathrm{C}$, followed by 2 days of incubation at room temperature. Colonies were suspended in PBS and spread onto $1.7 \%$ agarose slides. Samples were analyzed for green fluorescence in a Leica DM6000 B upright microscope coupled to an Andor iXon + camera, using $1000 \times$ amplification and a fluorescein isothiocyanate (FITC) filter. The images were analyzed using the MetaMorph Microscopy Automation and Image Analysis Software (Molecular Devices).

\section{Site-Directed Mutagenesis of E. coli YtfE and Production of Proteins}

Site-directed mutants of E. coli YtfE were constructed on the template pET-YtfE (Justino et al., 2006) using the oligonucleotide pairs described in Supplementary Table 5, and the QuikChange II Site-Directed Mutagenesis Kit and protocol (Agilent Technologies). Reaction products were transformed in E. coli XL1-Blue competent cells (Agilent Technologies), and positive recombinant vectors were selected on agar-plates containing $30 \mu \mathrm{g} / \mathrm{mL}$ kanamycin. Primers were designed so that the YtfE codons for E159 and E125 were replaced by those of leucine, asparagine and aspartate. Also, cysteines C30 and C31 of YtfE were changed to alanines. The E. coli $y t f E$ gene encoding the C30AC31A mutagenized protein (designated as $\mathrm{YtfE}^{\mathrm{M}}$ ) was used 
to generate other mutants ( $\mathrm{YtfE}^{\mathrm{M}}-\mathrm{E} 125 \mathrm{~L}$ and $\mathrm{YtfE}^{\mathrm{M}}-\mathrm{E} 159 \mathrm{~L}$ ). Also, YtfE genes in which the codons for E159 and E125 were replaced by those of leucine, asparagine and aspartate were constructed. All plasmids were sequenced confirming the presence of the desired mutations and the absence of unwanted modifications. Table 1 summarizes the site-directed mutants of YtfE and $\mathrm{YtfE}^{\mathrm{M}}$ proteins constructed in this work.

YtfE wild-type and its mutant proteins were expressed in E. coli BL21(DE3) Gold (Agilent) by growing cells in M9 minimal medium supplemented with $20 \mathrm{mM}$ glucose and $0.1 \mu \mathrm{M} \mathrm{FeSO}_{4}$, under aerobic conditions and at $30^{\circ} \mathrm{C}$, to an $\mathrm{OD}_{600}$ of 0.3 . At this point, $200 \mu \mathrm{M}$ IPTG was added and expression was induced for $7 \mathrm{~h}$. Cells were disrupted in a French Press, centrifuged and the soluble extracts loaded sequentially on a Q-Sepharose High-Performance and a Superdex S-75 gel filtration columns, coupled to an AKTA Purifier 10 FPLC System (GE Healthcare) following the protocol previously described (Justino et al., 2006). Proteins were judged pure by SDS-PAGE, and the iron content was determined by the 2,4,6-tripyridyl-S-triazine (TPTZ) method (Fischer and Price, 1964).

\section{Iron Binding Assays}

To determine the Fe(III) dissociation constant and the rate of iron release from YtfE wild-type and site-directed mutants, the as-isolated proteins $(25 \mu \mathrm{M})$ were incubated under aerobic conditions with the ferric chelator desferrioxamine (5-1000 $\mu \mathrm{M}$, Sigma; Nobre et al., 2014). Reaction mixtures were prepared in $20 \mathrm{mM}$ Tris- $\mathrm{HCl}$ pH 7.5 buffer with $150 \mathrm{mM} \mathrm{NaCl}$, incubated at room temperature for $24 \mathrm{~h}$ to attain thermodynamic equilibrium and analyzed by UV-visible spectroscopy in a Shimadzu UV-1700 spectrophotometer.

The $\mathrm{K}_{\mathrm{d}}, Y t f E$-chelator, defined by $\mathrm{K}_{\mathrm{d}}, Y t f E$-chelator $=$ [YtfE$\mathrm{Fe}][$ Chelator] $/([$ Chelator-Fe][YtfE $])$, is the dissociation constant of iron from YtfE in the presence of the competing chelator (Nobre et al., 2014). The constant was determined with GraphPad Prism version 5 for Windows (GraphPad Software, San Diego, CA, United States, www.graphpad.com) using the equation for a saturation binding experiment with one specific binding site: $\mathrm{Y}=\mathrm{B}_{\max }{ }^{*} \mathrm{X} /\left(\mathrm{K}_{d, Y t f E-\text { chelator }}+\mathrm{X}\right)$, where $\mathrm{X}$ is the concentration of the chelator, $\mathrm{Y}$ the percentage of iron atoms released and $\mathrm{B}_{\max }$ the maximum percentage of iron atoms released in relation to the total number of iron atoms in the isolated protein. The extinction coefficient used for desferrioxamine-Fe(III) was $2.9 \times 10^{3} \mathrm{M}^{-1} \mathrm{~cm}^{-1}$. The initial rate values $\left(\mathrm{V}_{0}\right)$ for iron dissociation were calculated from the linear part of the curves fit to the experimental results, considering only the first hours of the reaction.

\section{Assembly of Fe-S Clusters in IscU Promoted by YtfE}

Escherichia coli M15:pREP4 cells (Quiagen) harboring plasmids pQE30-(His)6-IscS and pQE60-IscU-(His)6 were used to produce E. coli IscS and apo-IscU proteins with a His-tag at the $N$ - and $C$-terminal, respectively, and were purified as previously described (Tokumoto et al., 2002; Nobre et al., 2014). Formation of the $\mathrm{Fe}-\mathrm{S}$ cluster in IscU promoted by YtfE wild-type and mutants was evaluated in reaction mixtures containing apo-IscU (50 $\mu \mathrm{M})$, IscS $(4 \mu \mathrm{M})$, DTT $(4 \mathrm{mM})$, and YtfE $(150 \mu \mathrm{M})$, in $20 \mathrm{mM}$ Tris-HCl pH 7.5 buffer supplemented with $150 \mathrm{mM}$ $\mathrm{NaCl}$. In all cases, addition of L-cysteine $(3 \mathrm{mM})$ initiated the reaction performed under anaerobic conditions at room temperature for $1 \mathrm{~h}$, and the process was analyzed by visible spectroscopy in a Shimadzu UV-1700 spectrophotometer. Reaction mixtures that contained all components except YtfE served as controls.

\section{Crystallization and X-ray Diffraction Data Collection and Analysis}

All the proteins used for crystallization procedures were purified in the buffer Tris- $\mathrm{HCl} 20 \mathrm{mM} \mathrm{pH} 7.5$ supplemented with $150 \mathrm{mM} \mathrm{NaCl}$ and concentrated to $20 \mathrm{mg} / \mathrm{mL}$. XRL hanging drop 24-well MD3-11 plates (Molecular Dimensions) were used for protein crystallization, with $0.5 \mathrm{~mL}$ of reservoir in each well. All experiments were done at room temperature.

Crystals of E. coli $\mathrm{YtfE}^{\mathrm{M}}$ and $\mathrm{YtfE}^{\mathrm{M}}$-E125L were prepared by the hanging drop vapor diffusion method using 1.0:0.8:0.2 $\mu \mathrm{L}$ mixtures of protein-reservoir-additive solutions. The reservoir solutions were constituted by Tris- $\mathrm{HCl} 0.1 \mathrm{M} \mathrm{pH} 7.5,25 \%$ PEG $4 \mathrm{~K}$ and $0.2 \mathrm{M} \mathrm{MgCl}_{2}$ plus the additive $\mathrm{NaCl}$ (2 M; Hampton Research). Crystals of $\mathrm{YtfE}^{\mathrm{M}}$-E159L were prepared by the hanging drop vapor diffusion method using 1:1 $\mu \mathrm{L}$ mixtures of proteinreservoir solution, constituted by Tris- $\mathrm{HCl} 0.1 \mathrm{M} \mathrm{pH} \mathrm{8.5,30 \%}$ PEG $4 \mathrm{~K}$, and $0.2 \mathrm{M} \mathrm{MgCl}$. These crystals were optimized by the micro-seeding technique (using crystals of $\mathrm{YtfE}^{\mathrm{M}}-\mathrm{E} 125 \mathrm{~L}$ to obtain the first crystals) plus streak-seeding (using the first crystals of $\mathrm{YtfE}^{\mathrm{M}}$-E159L).

Needle-shaped crystals were observed within a few minutes after plate set-up and grew to their maximal dimensions in 34 days. Crystals were harvested, immersed in a cryoprotectant solution with the same composition as the reservoir solution supplemented with $25 \%(\mathrm{v} / \mathrm{v})$ glycerol, flash frozen in liquid nitrogen, and sent to a synchrotron beamline for data collection. The $\mathrm{YtfE}^{\mathrm{M}}$ data set was collected at ALBA beamline XALOC (Juanhuix et al., 2014; Barcelona, Spain). The YtfE ${ }^{\mathrm{M}}$-E125L data set was measured at Diamond Light Source beamline I04 (DLS, Didcot, United Kingdom). The $\mathrm{YtfE}^{\mathrm{M}}$-E159L data set was recorded at beamline ID30A-3 of the European Synchrotron Radiation Facility (ESRF, Grenoble, France). The data collection and processing statistics are shown in Supplementary Table 1.

The $\mathrm{YtfE}^{\mathrm{M}}$ and $\mathrm{YtfE}^{\mathrm{M}}$-E125L data sets were integrated and scaled with XDS (Kabsch, 2010), analyzed with POINTLESS (Evans, 2006), and merged with AIMLESS (Evans and Murshudov, 2013). The YtfE ${ }^{\mathrm{M}}$-E159L data set was integrated and scaled with XDS and AutoPROC (Vonrhein et al., 2011), analyzed with POINTLESS and scaled and merged with STARANISO (Tickle et al., 2018) and AIMLESS. The structures were solved by molecular replacement with PHASER (McCoy, 2006) via the CCP4 (Potterton et al., 2003) Graphics User Interface using the previously reported E. coli YtfE structure (PDB 5FNN; Lo et al., 2016) as the search model. Following an initial refinement with REFMAC (Murshudov et al., 2011) the models were improved by sequential cycles of correction using COOT (Emsley et al., 2010). 
Refinement proceeded with PHENIX (Adams et al., 2010) in five macrocycle steps, with refinement of positional coordinates, individual isotropic atomic displacement parameters for all nonhydrogen atoms, occupancies and using non-crystallographic symmetry restraints for the two independent molecules in the asymmetric unit. Hydrogen atoms were added to the structural models and included in the refinement in calculated positions. The examination and editing of the models between refinements was done with COOT against $\sigma_{A}$-weighted $2\left|\mathrm{~F}_{o}\right|-\left|\mathrm{F}_{\mathrm{c}}\right|$ and $\left|\mathrm{F}_{o}\right|-\left|\mathrm{F}_{\mathrm{c}}\right|$ electron density maps. TLS (translation-librationscrew) rigid body refinement of atomic displacement parameters was carried out for all structures, followed by refinement of individual isotropic B-factors. Two TLS groups were used for each chain, corresponding approximately to the two protein domains. Water molecules were added with PHENIX and verified with COOT. MOLPROBITY (Chen et al., 2010) was used to inspect the model geometry together with the validation tools available in COOT. The refinement statistics are included in Supplementary Table 2. Structure figures were created using the PyMOL Molecular Graphics System, Version 2.3.4 Open Source (DeLano, 2020).

The tunnels were computed using the software MOLE 2.5 (Sehnal et al., 2013), with default parameters (probe radius of $3 \AA$, interior threshold of $1.25 \AA$, minimum depth of $5 \AA$, and bottleneck radius of $1.25 \AA$ ) for the $\mathrm{YtfE}^{\mathrm{M}}$ structure. For $\mathrm{YtfE}^{\mathrm{M}}$-E159L the parameters were optimized (interior threshold of $1.18 \AA$ and bottleneck radius of $1.13 \AA$ ). The indicated width of the tunnel at each point corresponds to the empty space between the Van der Waals spheres representing the atoms of the amino acid residues lining the tunnel.

The final atomic coordinates and experimental structure factors were deposited in the Worldwide Protein Data Bank (Burley et al., 2019) with the accession codes 7BHA, 7BHB, and $7 \mathrm{BHC}$ for the $\mathrm{YtfE}^{\mathrm{M}}$, $\mathrm{YtfE}^{\mathrm{M}}-\mathrm{E} 159 \mathrm{~L}$, and $\mathrm{YtfE}^{\mathrm{M}}-\mathrm{E} 125 \mathrm{~L}$ structures, respectively. Data collection and refinement statistics are summarized in Supplementary Tables 1, 2.

\section{DATA AVAILABILITY STATEMENT}

The datasets presented in this study can be found in online repositories. The names of the repository/repositories and accession number(s) can be found below: PDB accession numbers: 7BHA, 7BHB, and 7BHC. https://doi.org/10.2210/ pdb7BHA/pdb; https://doi.org/10.2210/pdb7BHB/pdb; and https://doi.org/10.2210/pdb7BHC/pdb.

\section{REFERENCES}

Adams, P. D., Afonine, P. V., Bunkóczi, G., Chen, V. B., Davis, I. W., Echols, N., et al. (2010). PHENIX: a comprehensive Python-based system for macromolecular structure solution. Acta Crystallogr. Sect. D Biol. Crystallogr. 66, 213-221. doi: 10.1107/S0907444909052925

Alvarez-Carreño, C., Alva, V., Becerra, A., and Lazcano, A. (2018). Structure, function and evolution of the hemerythrin-like domain superfamily. Protein Sci. 27, 848-860. doi: 10.1002/pro.3374

\section{AUTHOR CONTRIBUTIONS}

LOS produced the proteins and performed biochemical experiments, protein-protein interactions studies, and crystal production. CVR, PM, and LOS collected and analyzed the crystallographic data. LMS, LOS, and CVR wrote the manuscript with contributions from PM. LMS supervised the work and designed the study. All authors contributed to the article and approved the submitted version.

\section{FUNDING}

This work was financially supported by Fundação para a Ciência e Tecnologia (Portugal) through fellowship SFRH/BD/118545/2016 (LOS) and R\&D unit LISBOA-010145-FEDER007660 (MostMicro) co-funded by FCT/MCTES and FEDER funds under the PT2020 Partnership Agreement. This work was partially supported by PPBI - Portuguese Platform of BioImaging (PPBI-POCI-01-0145-FEDER-022122) co-funded by national funds from OE - "Orçamento de Estado" and by European funds from FEDER - "Fundo Europeu de Desenvolvimento Regional." We also acknowledge funding from the European Union's Horizon 2020 Research and Innovation Program under grant agreement no. 810856.

\section{ACKNOWLEDGMENTS}

We thank Lígia S. Nobre and Joana M. Baptista for contribution at the initial stage of the work, and Cláudia S. Freitas for technical support. We also thank Professor Miguel Teixeira of ITQBNOVA for critical reading of the manuscript. We thank the XALOC staff and floor coordinators at the synchrotron ALBA for the $\mathrm{YtfE}^{\mathrm{M}}$ data collection. We acknowledge the ESRF for provision of synchrotron radiation facilities and we would like to thank Gianluca Santoni for assistance using the beamline ID30A-3 for the $\mathrm{YtfE}^{\mathrm{M}}$-E159L data collection. We also thank Diamond Light Source for beamtime and the staff of beamline I04 for assistance with crystal testing and data collection of $\mathrm{YtfE}^{\mathrm{M}}-\mathrm{E} 125 \mathrm{~L}$.

\section{SUPPLEMENTARY MATERIAL}

The Supplementary Material for this article can be found online at: https://www.frontiersin.org/articles/10.3389/fmicb. 2021.670681/full\#supplementary-material

Baker, N. A., Sept, D., Joseph, S., Holst, M. J., and McCammon, J. A. (2001). Electrostatics of nanosystems: application to microtubules and the ribosome. Proc. Natl. Acad. Sci. U.S.A. 98, 10037-10041. doi: 10.1073/pnas.181342398

Blanc, B., Gerez, C., and Ollagnier de Choudens, S. (2015). Assembly of Fe/S proteins in bacterial systems. Biochemistry of the bacterial ISC system. Biochim. Biophys. Acta - Mol. Cell Res. 1853, 1436-1447. doi: 10.1016/j.bbamcr.2014.12. 009

Brunskill, E. W., De Jonge, B. L. M. M., and Bayles, K. W. (1997). The Staphylococcus aureus scdA gene: a novel locus that affects cell division and 
morphogenesis. Microbiology 143, 2877-2882. doi: 10.1099/00221287-143-92877

Burley, S. K., Berman, H. M., Bhikadiya, C., Bi, C., Chen, L., Costanzo, L., et al. (2019). Protein Data Bank: the single global archive for 3D macromolecular structure data. Nucleic Acids Res. 47, D520-D528. doi: 10.1093/nar/gky949

Chang, W., Small, D. A., Toghrol, F., and Bentley, W. E. (2006). Global transcriptorne analysis of Staphylococcus aureus response to hydrogen peroxide. J. Bacteriol. 188, 1648-1659. doi: 10.1128/JB.188.4.1648-1659.2006

Chen, V. B., Arendall, W. B., Headd, J. J., Keedy, D. A., Immormino, R. M., Kapral, G. J., et al. (2010). MolProbity: all-atom structure validation for macromolecular crystallography. Acta Crystallogr. Sect. D Biol. Crystallogr. 66, 12-21. doi: 10. 1107/S0907444909042073

Davis, K. M., Krupp, J., Clark, S., and Isberg, R. R. (2019). Iron-Sulfur cluster repair contributes to Yersinia pseudotuberculosis survival within deep tissues. Infect. Immun. 87, e00533-e00619. doi: 10.1128/IAI.00533-19

DeLano, W. L. (2020). The PyMOL Molecular Graphics System, Version 2.3. New York, NY: Schrödinger LLC.

Ding, H., and Clark, R. J. (2004). Characterization of iron binding in IscA, an ancient iron-sulphur cluster assembly protein. Biochem. J. 379, 433-440. doi: 10.1042/BJ20031702

Dolinsky, T. J., Nielsen, J. E., McCammon, J. A., and Baker, N. A. (2004). PDB2PQR: an automated pipeline for the setup of Poisson-Boltzmann electrostatics calculations. Nucleic Acids Res. 32, W665-W667. doi: 10.1093/ nar/gkh381

Ebrahimi, K. H., Hagedoorn, P.-L., and Hagen, W. R. (2015). Unity in the biochemistry of the iron-storage proteins ferritin and bacterioferritin. Chem. Rev. 115, 295-326. doi: 10.1021/cr5004908

Emsley, P., Lohkamp, B., Scott, W. G., and Cowtan, K. (2010). Features and development of Coot. Acta Crystallogr. Sect. D Biol. Crystallogr. 66, 486-501. doi: $10.1107 / S 0907444910007493$

Evans, P. (2006). Scaling and assessment of data quality. Acta Crystallogr. Sect. D: Biol. Crystallogr. (International Union of Crystallography) 62, 72-82. doi: $10.1107 / \mathrm{S} 0907444905036693$

Evans, P. R., and Murshudov, G. N. (2013). How good are my data and what is the resolution? Acta Crystallogr. Sect. D Biol. Crystallogr. 69, 1204-1214. doi: $10.1107 /$ S0907444913000061

Fischer, D. S., and Price, D. C. (1964). A simple serum iron method using the new sensitive chromogen tripyridyl-s-triazine. Clin. Chem. 10, 21-31. doi: 10.1093/ clinchem/10.1.21

Harrington, J. C., Wong, S. M. S., Rosadini, C. V., Garifulin, O., Boyartchuk, V., and Akerley, B. J. (2009). Resistance of Haemophilus influenzae to reactive nitrogen donors and gamma interferon-stimulated macrophages requires the formatedependent nitrite reductase regulator-activated $y t f E$ gene. Infect. Immun. 77, 1945-1958. doi: 10.1128/IAI.01365-08

Juanhuix, J., Gil-Ortiz, F., Cuní, G., Colldelram, C., Nicolás, J., Lidón, J., et al. (2014). Developments in optics and performance at BL13-XALOC, the macromolecular crystallography beamline at the Alba Synchrotron. J. Synchrotron Radiat. 21, 679-689. doi: 10.1107/S160057751400825X

Justino, M. C., Almeida, C. C., Gonçalves, V. L., Teixeira, M., and Saraiva, L. M. (2006). Escherichia coli YtfE is a di-iron protein with an important function in assembly of iron-sulphur clusters. FEMS Microbiol. Lett. 257, 278-284. doi: 10.1111/j.1574-6968.2006.00179.x

Justino, M. C., Baptista, J. M., and Saraiva, L. M. (2009). Di-iron proteins of the Ric family are involved in iron-sulfur cluster repair. Biometals 22, 99-108. doi: 10.1007/s10534-008-9191-2

Justino, M. C., Vicente, J. B., Teixeira, M., and Saraiva, L. M. (2005). New genes implicated in the protection of anaerobically grown Escherichia coli against nitric oxide. J. Biol. Chem. 280, 2636-2643. doi: 10.1074/jbc.M411070200

Kabsch, W. (2010). XDS. Acta Crystallogr. D. Biol. Crystallogr. 66, 125-132. doi: $10.1107 /$ S0907444909047337

Karimova, G., Pidoux, J., Ullmann, A., and Ladant, D. (1998). A bacterial twohybrid system based on a reconstituted signal transduction pathway. Proc. Natl. Acad. Sci. U.S.A. 95, 5752-5756. doi: 10.1073/pnas.95.10.5752

Kato, S. I., Mihara, H., Kurihara, T., Takahashi, Y., Tokumoto, U., Yoshimura, T., et al. (2002). Cys-328 of IscS and Cys-63 of IscU are the sites of disulfide bridge formation in a covalently bound IscS/IscU complex: implications for the mechanism of iron-sulfur cluster assembly. Proc. Natl. Acad. Sci. U.S.A. 99, 5948-5952. doi: 10.1073/pnas.082123599
Kim, J. H., Bothe, J. R., Alderson, T. R., and Markley, J. L. (2015). Tangled web of interactions among proteins involved in iron-sulfur cluster assembly as unraveled by NMR, SAXS, chemical crosslinking, and functional studies. Biochim. Biophys. Acta - Mol. Cell Res. 1853, 1416-1428. doi: 10.1016/j.bbamcr. 2014.11.020

Kim, J. H., Fuzéry, A. K., Tonelli, M., Ta, D. T., Westler, W. M., Vickery, L. E., et al. (2009). Structure and dynamics of the iron-sulfur cluster assembly scaffold protein IscU and Its interaction with the cochaperone HscB. Biochemistry 48, 6062-6071. doi: 10.1021/bi9002277

Layer, G., Ollagnier-De Choudens, S., Sanakis, Y., and Fontecave, M. (2006). Ironsulfur cluster biosynthesis: characterization of Escherichia coli CyaY as an iron donor for the assembly of [2Fe-2S] clusters in the scaffold IscU. J. Biol. Chem. 281, 16256-16263. doi: 10.1074/jbc.M513569200

Lo, F.-C., Hsieh, C.-C., Maestre-Reyna, M., Chen, C.-Y., Ko, T.-P., Horng, Y.-C., et al. (2016). Crystal structure analysis of the repair of iron centers protein YtfE and its interaction with NO. Chem. - A Eur. J. 22, 9768-9776. doi: 10.1002/ chem. 201600990

Lu, J., Bi, B., Lai, W., and Chen, H. (2019). Origin of nitric oxide reduction activity in flavo-diiron NO reductase: key roles of the second coordination sphere. Angew. Chemie Int. Ed. 58, 3795-3799. doi: 10.1002/anie.20181 2343

Lu, J., Yang, J., Tan, G., and Ding, H. (2008). Complementary roles of SufA and IscA in the biogenesis of iron-sulfur clusters in Escherichia coli. Biochem. J. 409, 535-543. doi: 10.1042/BJ20071166

Macedo, S., Romão, C. V., Mitchell, E., Matias, P. M., Liu, M. Y., Xavier, A. V., et al. (2003). The nature of the di-iron site in the bacterio-ferritin from Desulfovibrio desulfuricans. Nat. Struct. Biol. 10, 285-290. doi: 10.1038/nsb909

McCoy, A. J. (2006). Solving structures of protein complexes by molecular replacement with Phaser. Acta Crystallogr. Sect. D: Biol. Crystallogr. (International Union of Crystallography) 63(Pt 1), 32-41. doi: 10.1107/ S0907444906045975

Murshudov, G. N., Skubák, P., Lebedev, A. A., Pannu, N. S., Steiner, R. A., Nicholls, R. A., et al. (2011). REFMAC5 for the refinement of macromolecular crystal structures. Acta Crystallogr. Sect. D Biol. Crystallogr. 67, 355-367. doi: 10.1107/ S0907444911001314

Nobre, L. S., Garcia-Serres, R., Todorovic, S., Hildebrandt, P., Teixeira, M., Latour, J.-M., et al. (2014). Escherichia coli RIC is able to donate iron to iron-sulfur clusters. PLoS One 9:e95222. doi: 10.1371/journal.pone.0095222

Nobre, L. S., Lousa, D., Pacheco, I., Soares, C. M., Teixeira, M., and Saraiva, L. M. (2015). Insights into the structure of the diiron site of RIC from Escherichia coli. FEBS Lett. 589, 426-431. doi: 10.1016/j.febslet.2014.12.028

Nobre, L. S., Meloni, D., Teixeira, M., Viscogliosi, E., and Saraiva, L. M. (2016). Trichomonas vaginalis repair of iron centres proteins: the different role of two paralogs. Protist 167, 222-233. doi: 10.1016/j.protis.2016.03.001

Overton, T. W., Justino, M. C., Li, Y., Baptista, J. M., Melo, A. M. P., Cole, J. A., et al. (2008). Widespread distribution in pathogenic bacteria of di-iron proteins that repair oxidative and nitrosative damage to iron-sulfur centers. J. Bacteriol. 190, 2004-2013. doi: 10.1128/JB.01733-07

Potterton, E., Briggs, P., Turkenburg, M., and Dodson, E. (2003). A graphical user interface to the CCP4 program suite. Acta Crystallogr. - Sect. D Biol. Crystallogr. 59, 1131-1137. doi: 10.1107/S0907444903008126

Prischi, F., Pastore, C., Carroni, M., Iannuzzi, C., Adinolfi, S., Temussi, P., et al. (2010). Of the vulnerability of orphan complex proteins: the case study of the E. coli IscU and IscS proteins. Protein Expr. Purif. 73, 161-166. doi: 10.1016/J. PEP.2010.05.003

Roche, B., Aussel, L., Ezraty, B., Mandin, P., Py, B., and Barras, F. (2013). Iron/sulfur proteins biogenesis in prokaryotes: formation, regulation and diversity. Biochim. Biophys. Acta - Bioenerg. 1827, 455-469. doi: 10.1016/j. bbabio.2012.12.010

Roche, B., Huguenot, A., Barras, F., and Py, B. (2015). The iron-binding CyaY and IscX proteins assist the ISC-catalyzed Fe-S biogenesis in Escherichia coli. Mol. Microbiol. 95, 605-623. doi: 10.1111/mmi.12888

Rui, H., Rivera, M., and Im, W. (2012). Protein dynamics and ion traffic in bacterioferritin. Biochemistry 51, 9900-9910. doi: 10.1021/bi3013388

Schwartz, C. J., Djaman, O., Imlay, J. A., and Kiley, P. J. (2000). The cysteine desulfurase, IscS, has a major role in in vivo Fe-S cluster formation in Escherichia coli. Proc. Natl. Acad. Sci. U.S.A. 97, 9009-9014. doi: 10.1073/pnas. 160261497 
Sehnal, D., Vařeková, R. S., Berka, K., Pravda, L., Navrátilová, V., Banáš, P., et al. (2013). MOLE 2.0: advanced approach for analysis of biomacromolecular channels. J. Cheminform. 5:39. doi: 10.1186/1758-2946-5-39

Silva, L. O., Nobre, L. S., Mil-Homens, D., Fialho, A., and Saraiva, L. M. (2018). Repair of Iron Centers RIC protein contributes to the virulence of Staphylococcus aureus. Virulence 9, 312-317. doi: 10.1080/21505594.2017. 1389829

Silva, L. S. O., Baptista, J. M., Batley, C., Andrews, S. C., and Saraiva, L. M. (2018). The Di-iron RIC protein (YtfE) of Escherichia coli interacts with the DNAbinding protein from starved cells (Dps) to diminish RIC protein-mediated redox stress. J. Bacteriol. 200, e00527-e00618. doi: 10.1128/JB.00527-18

Skovran, E., Lauhon, C. T., and Downs, D. M. (2004). Lack of YggX results in chronic oxidative stress and uncovers subtle defects in Fe-S cluster metabolism in Salmonella enterica. J. Bacteriol. 186, 7626-7634. doi: 10.1128/JB.186.22. 7626-7634.2004

Swartz, L., Kuchinskas, M., Li, H., Poulos, T. L., and Lanzilotta, W. N. (2006). Redox-dependent structural changes in the Azotobacter vinelandii bacterioferritin: new insights into the ferroxidase and iron transport mechanism. Biochemistry 45, 4421-4428. doi: 10.1021/bi060146w

Tan, G., Lu, J., Bitoun, J. P., Huang, H., and Ding, H. (2009). IscA/SufA paralogues are required for the [ $4 \mathrm{Fe}-4 \mathrm{~S}]$ cluster assembly in enzymes of multiple physiological pathways in Escherichia coli under aerobic growth conditions. Biochem. J. 420, 463-472. doi: 10.1042/BJ20090206

Tatur, J., Hagen, W. R., and Matias, P. M. (2007). Crystal structure of the ferritin from the hyperthermophilic archaeal anaerobe Pyrococcus furiosus. J. Biol. Inorg. Chem. 12, 615-630. doi: 10.1007/s00775-007-0212-3

Tickle, I. J., Flensburg, C., Keller, P., Paciorek, W., Sharff, A., Vonrhein, C., et al. (2018). STARANISO. Cambridge: Global Phasing Ltd.

Todorovic, S., Justino, M. C., Wellenreuther, G., Hildebrandt, P., Murgida, D. H., Meyer-Klaucke, W., et al. (2008). Iron-sulfur repair YtfE protein from Escherichia coli: structural characterization of the di-iron center. J. Biol. Inorg. Chem. 13, 765-770. doi: 10.1007/s00775-008-0362-y
Tokumoto, U., Nomura, S., Minami, Y., Mihara, H., Kato, S. S.-I., Kurihara, T., et al. (2002). Network of protein-protein interactions among iron-sulfur cluster assembly proteins in Escherichia coli. J. Biochem. 131, 713-719. doi: 10.1093/ oxfordjournals.jbchem.a003156

Vinella, D., Brochier-Armanet, C., Loiseau, L., Talla, E., and Barras, F. (2009). IronSulfur (Fe/S) protein biogenesis: phylogenomic and genetic studies of A-Type carriers. PLoS Genet. 5:e1000497. doi: 10.1371/journal.pgen.1000497

Vonrhein, C., Flensburg, C., Keller, P., Sharff, A., Smart, O., Paciorek, W., et al. (2011). Data processing and analysis with the autoPROC toolbox. Acta Crystallogr. Sect. D Biol. Crystallogr. 67, 293-302. doi: 10.1107/ S0907444911007773

Weeratunga, S. K., Lovell, S., Yao, H., Battaile, K. P., Fischer, C. J., Gee, C. E., et al. (2010). Structural studies of bacterioferritin B from Pseudomonas aeruginosa suggest a gating mechanism for iron uptake via the ferroxidase center. Biochemistry 49, 1160-1175. doi: 10.1021/bi9015204

Wilson, C. G. M., Magliery, T. J., and Regan, L. (2004). Detecting protein-protein interactions with GFP-fragment reassembly. Nat. Methods 1, 255-262. doi: 10. 1038/nmeth1204-255

Zheng, L., Cash, V. L., Flint, D. H., and Dean, D. R. (1998). Assembly of iron-sulfur clusters. Identification of an iscSUA-hscBA-fdx gene cluster from Azotobacter vinelandii. J. Biol. Chem. 273, 13264-13272. doi: 10.1074/jbc.273.21.13264

Conflict of Interest: The authors declare that the research was conducted in the absence of any commercial or financial relationships that could be construed as a potential conflict of interest.

Copyright $\odot 2021$ Silva, Matias, Romão and Saraiva. This is an open-access article distributed under the terms of the Creative Commons Attribution License (CC BY). The use, distribution or reproduction in other forums is permitted, provided the original author(s) and the copyright owner(s) are credited and that the original publication in this journal is cited, in accordance with accepted academic practice. No use, distribution or reproduction is permitted which does not comply with these terms. 